\title{
Valor e Significado da Vivência no Primeiro Ano do Curso de Medicina: Apreciação de Aprendizado Pessoal e Contexto em uma Série Histórica
}

PALAVRAS-CHAVE:

-Educação de Graduação em Medicina;

-Estudantes de Medicina;

-Aprendizagem;

-Avaliação Educacional.

\section{KEY-WORDS:}

-Education, Medical,

Undergraduate;

-Students, Medical;

-Learning;

-Educational Measurement.

Recebido em: 09/01/2007

Aprovado em: 12/06/2007

\section{Value and Meaning of the First-Year Learning Experience in a Medical Program: An Appraisal of Personal Learning and Context in a Historic Series}

Dejano T. Sobral ${ }^{1}$

\section{RESUMO}

Os propósitos do trabalho foram examinar as percepções dos alunos sobre a vivência inicial do curso de Medicina, mediante o Questionário de Valoração do Curso (QVC), bem como analisar relações entre índices do QVC e medidas de atributos pessoais e sucesso acadêmico. O estudo envolveu 609 alunos numa década. Os resultados revelaram relativa estabilidade dos escores do QVC em turmas consecutivas e diferenças significantes entre os alunos agrupados por sexo, naturalidade, categoria de preferência por carreira e semestre de acesso ao curso. Verificaram-se, também, associações significantes entre escores do QVC e medidas de autoconfiança, intenção de aprender, rendimento cognitivo e atividade de monitoria em quatro semestres seguidos. Testes do Modelo Linear Geral revelaram quatro fatores independentes principais na explicação da variabilidade do escore do QVC: intenção de aprender, autoconfiança, preferência por carreira e semestre de acesso. A discussão examina os achados em conexão com noções de contexto interno do aprendiz e ambiência educacional. Concluindo, as respostas ao QVC refletem a perspectiva de valoração do aprendizado pessoal na interação com as dimensões do contexto educacional. O instrumento tem, assim, utilidade no acompanhamento pedagógico da iniciação profissional.

\begin{abstract}
The purpose of this study was to appraise how medical students perceive their first-year experiences in the medical course using an adapted version of the Course Valuing Inventory (CVI), and to analyse the relationships between the CVI scores and the students' individual attributes and academic achievements. The study involved 609 students and covered a period of ten years. The results showed relative stability of CVI scores in consecutive classes and significant differences among students when grouped by sex, place of birth, career choice, and term of enrolment in the program. Other findings were the significant relationships between CVI score and self-confidence, willingness to learn and cognitive achievement, and peer tutoring activity during four consecutive terms. GLM tests (between subjects and effects) identified four principal independent factors to explain the CVI-score variability: willingness to learn, self-confidence, career choice and term of enrolment. The discussion appraises the findings in relation to concepts of
\end{abstract}


the students, internal context and educational context. In conclusion, CVI responses reflect a perspective of valuing personal learning in interaction with dimensions of the educational context. Accordingly, this tool can be considered useful for the pedagogical supervision of medical students' professional initiation.

\section{INTRODUÇÃO}

Em que medida as percepções da qualidade da vivência no primeiro ano de estudos são influenciadas por fatores pessoais e afetam o progresso acadêmico dos alunos de Medicina? Os trabalhos de Genn ${ }^{1,2}$ sugerem que percepções da qualidade da ambiência educacional influenciam as respostas dos alunos aos processos de ensino e aprendizagem, afetando a adaptação motivacional dos aprendizes e o rendimento acadêmico.

A questão tem relevância especial para a vivência do primeiro ano universitário. Ao ingressar no curso, em contraponto ao impacto da transição, a grande maioria dos alunos de Medicina tem expectativas quanto à preparação para a prática médica, mas não tanto quanto à atividade acadêmica em si. Tais expectativas já se denotam pelas razões de influência na escolha da carreira, em que sobressaem fatores de altruísmo e busca de desafio profissional, acima de injunções ou recompensas externas (dados não publicados). Ao longo do primeiro ano, componentes diversos, formais e informais, das oportunidades e limitações da aprendizagem para a prática são revelados, os quais incluem principalmente as representações explícitas e implícitas do currículo que se expressam na caracterização da ambiência educacional.

Como fazer uma apreciação do impacto educacional da iniciação no curso a partir da perspectiva dos aprendizes? Essa vertente da avaliação educacional tem sido examinada por muitos autores ao longo das últimas décadas. Sob uma ótica humanista, Nehari e Bender propuseram um modelo conceitual para aferir a valoração de uma vivência de aprendizagem e seus efeitos no aprendiz, sugerindo que esses desfechos educacionais estariam relacionados às percepções de aprendizado nos domínios de conteúdo cognitivo, afetivo-pessoal e comportamental ${ }^{3}$. Eles desenvolveram um instrumento - Course Valuing Inventory - para medir o valor e o significado de um curso em conjunção com as percepções de aprendizado nos domínios referidos. Naquele trabalho, os autores indicaram que tanto o nível educacional quanto a expertise dos aprendizes influenciavam suas percepções do valor de um curso específico, segundo a aferição proporcionada pelo inventário. Nehari e Bender sugeriram o uso do instrumento como uma medida dos esforços educacionais e dos efeitos de um curso nos alunos, em termos de mudança e crescimento ${ }^{3}$.

Outros trabalhos, na área da educação médica, mostraram a utilidade do inventário de Nehari e Bender para apreciação da valia de uma vivência de aprendizagem, no contexto tanto de disciplina isolada quanto de estrutura curricular mais ampla ${ }^{4,5}$. Tais trabalhos utilizaram uma versão em português do instrumento original, denominada doravante Questionário de Valoração do Curso (QVC). Em particular, as respostas dos alunos ao QVC em situações educacionais diversas permitiram diferenciar efeitos de distintos enfoques de ensino e aprendizagem nos índices do instrumento.

Um estudo mais recente reforçou a validade de construto desta versão do instrumento ${ }^{6}$. Em especial, evidenciou-se a validade concorrente do questionário, expressa na correlação importante e significativa entre o escore total do Dreem (Dundee Ready Education Environment Measure) - uma medida da qualidade da ambiência educacional7 - e dimensões críticas do QVC. Adicionalmente, verificou-se que o grau de positividade nas respostas ao QVC era direta e significantemente associado aos níveis de motivação autônoma, orientação significativa e reflexão na aprendizagem dos alunos. Esses achados suscitaram a hipótese de que as respostas ao QVC repercutem a configuração de aprendizagem pessoal, além da percepção da qualidade da ambiência educacional ou vivência curricular no sentido amplo.

A justificativa para o presente estudo foi a expectativa de confirmar e estender os achados anteriores, considerando alunados de turmas consecutivas a partir da implantação do acompanhamento curricular 2/88 no curso de Medicina da Universidade de Brasília. Dois propósitos foram estabelecidos: examinar as percepções da qualidade da vivência curricular inicial do curso, na forma detectada pelo QVC, bem como analisar as relações entre os índices do QVC e medidas de atributos pessoais e sucesso acadêmico dos alunos de Medicina. Este trabalho considera os seguintes objetivos:

- relatar os índices do QVC ao término do primeiro ano do curso de Medicina na série histórica de dez anos;

- determinar as relações entre o QVC e medidas de autoconfiança, motivação para aprender, preferência na escolha de carreira e rendimento cognitivo no primeiro ano;

- verificar a sensibilidade do QVC na predição da atividade de monitoria na fase pré-clínica, do rendimento cognitivo no início da fase clínica e da evasão ao término da graduação.

\section{MÉTODOS}

\section{Delineamento}

Trata-se de estudo retrospectivo baseado em dados compilados em dez anos de acompanhamento curricular, incluindo componentes transversal e prospectivo.

\section{Cenário}

O estudo foi realizado no contexto do curso de Medicina da Universidade de Brasília (UnB), cujo fluxo curricular abran- 
ge quatro semestres de disciplinas básicas, cinco semestres de disciplinas clínicas e três semestres de internato, desde 1988. O primeiro ano do curso compreende disciplinas obrigatórias de biologia celular, bioquímica e biofísica, anatomia e histologia, fisiologia e saúde coletiva, além de amplas oportunidades para cursar disciplinas optativas. A transição para a fase clínica ocorre no quinto período do fluxo curricular com a disciplina de semiologia.

\section{Participantes}

O critério de inclusão no estudo foi o acesso ao terceiro período do fluxo curricular, abrangendo, por amostragem consecutiva $^{8}$, cada aluno da primeira à vigésima turma semestral do novo acompanhamento curricular iniciado em 1988. Do total de 609 alunos, 55,7\% eram do sexo masculino e 45,5\% naturais do Distrito Federal (DF). A média de idade no ano de ingresso no curso era 19,4 anos (faixa 16 a 42). A grande maioria $\left(84,1 \%\right.$ ) ingressou na UnB por vestibular (do $1^{\circ}$ ou do $2^{\underline{o}}$ semestre) na própria universidade, e os restantes $15,9 \%$ por transferência de outras escolas ou acordo diplomático. O acesso ao curso no primeiro semestre alcançou 49,3\% dos participantes.

\section{Medidas e procedimentos}

Os dados do estudo derivam da aplicação do QVC e do inventário de características e expectativas do aprendiz, bem como do acompanhamento acadêmico dos participantes, em função de rendimento cognitivo, atividade de monitoria e conclusão do curso. A coleta de dados em cada turma foi realizada na primeira semana do terceiro período do fluxo curricular. Os dados foram obtidos com asseguramento expresso de confidencialidade pessoal. Cópias dos instrumentos utilizados estão disponíveis mediante solicitação para o endereço institucional de correspondência.

Questionário de Valoração do Curso. O formato do QVC que foi utilizado é reproduzido no anexo. Esse formato é uma versão adaptada do instrumento original - contido no trabalho de Nehari e Bender ${ }^{3}$ - em que a redação dos 36 itens foi modificada para atender à perspectiva da vivência curricular mais ampla e não apenas de uma única disciplina. $\mathrm{O}$ aluno responde a cada item numa escala de quatro pontos que afere o nível, negativo ou positivo, da reação ao enunciado do item. A soma das pontuações (ajustada para a polaridade invertida de 13 itens) constitui o escore total (faixa: 36-144). Este escore estabelece o nível de positividade (ou negatividade) da vivência no curso e reflete o valor e significado do aprendizado. $\mathrm{Na}$ amostra do estudo, a consistência interna do instrumento foi demonstrada pelos valores de três tipos de coeficiente: confi- abilidade (alfa de Cronbach $=0,928$ ), correlação item-total ajustada $(0,386$ a 0,614$)$ e correlação entre itens (média $=0,267$; faixa: 0,025-0,559). Uma análise de fatores (extração maximum likelihood) na amostra revelou essencialmente um fator maior explicando $29 \%$ da variância e que subentende a reafirmação do valor da vivência.

Inventário de características. Essa documentação forneceu dados sobre autoconfiança como aprendiz, motivação para aprender e preferência na escolha de futura carreira em medicina, além de estilo de aprendizagem (não analisado neste trabalho). Escalas analógicas visuais (100 mm), ancoradas nas extremas, foram utilizadas para duas medidas: autoconfiança como aprendiz e intenção de aprender. A primeira escala serve como indicador da percepção de eficácia pessoal na aprendizagem ${ }^{9}$, e a segunda como indicador de motivação imediata ou situacional.

Preferência por carreira. A expectativa pessoal sobre a futura carreira em medicina foi obtida mediante a identificação pelo participante da preferência por área de atuação médica dentre 35 opções agrupadas em oito categorias. Para efeito da análise, os participantes foram classificados em dois grupos, segundo a popularidade histórica da categoria de preferência inicial: o grupo maior incluiu preferência principal por Cirurgia ou Clínica Médica ou Pediatria; e o menor incluiu preferência por qualquer outra categoria de opção.

Registro de monitoria. Apurou-se, para cada participante, a ocorrência de atividade de monitoria por dois anos consecutivos (entre o segundo e o quinto período do curso). A realização de monitoria, nessa fase do curso, tem por objeto principal as disciplinas obrigatórias de anatomia, histologia, embriologia, fisiologia, farmacologia, patologia, imunologia, saúde coletiva, parasitologia e bioquímica e biofísica.

Rendimento cognitivo. O indicador utilizado foi o índice de rendimento acadêmico do aluno. Esse índice foi calculado para o primeiro ano e para o sexto período do curso, com base nas menções e no número de créditos das respectivas disciplinas obrigatórias. A faixa de pontuação do índice varia de zero a cinco.

\section{Análise}

Os textos de Fraenkel e Wallen ${ }^{10}$ e de Howell ${ }^{11}$ orientaram os planos e procedimentos de análise. Quatro procedimentos estatísticos principais foram utilizados: (a) testes $\mathbf{t}$ e análises de variância para aferir diferenças entre médias de grupos; (b) testes de qui-quadrado, para comparar características ou descritores entre grupos; (c) coeficientes de correlação para medir a associação entre pares de variáveis; (d) testes do Modelo Linear Geral, para verificar efeitos de 
distintos grupamentos de participantes no escore do QVC ajustado por co-variáveis. O nível de significância foi fixado em $\mathrm{P}=0,05$ (two-tailed). A perda parcial na análise incluiu 39 casos, dois por extravio de dados e 37 por evasão no período entre a inclusão no estudo e a graduação. A análise foi efetuada no programa SPSS 9.0.

\section{RESULTADOS}

A Tabela 1 resume as diferenças nas características dos participantes (atributos demográficos, preferência por carreira, semestre de acesso ao curso) quando agru- pados segundo o biênio de ingresso no curso. As diferenças entre as médias de idade dos grupos não foram significantes (Anova, $\mathrm{df}=4 / 604 ; \mathrm{F}=0,433 ; \mathrm{P}=0,748$ ). Não houve, também, diferenças significantes na distribuição de sexo, preferência por carreira ou semestre de acesso entre os grupos de ingresso (testes de qui-quadrado, menor $\mathrm{P}=0,421$ ). No entanto, observou-se tendência crescente significante na proporção de naturais do Distrito Federal ao longo dos cinco biênios de ingresso no curso (qui-quadrado para tendência linear $=12,5$; $\mathrm{df}=1 ; \mathrm{P}<0,001)$.

TABELA 1

Comparação de atributos demográficos (idade, sexo, naturalidade), semestre de acesso ao curso e preferência inicial por carreira entre alunos de Medicina agrupados segundo o biênio de ingresso no curso $(\mathrm{N}=609)$

\begin{tabular}{|c|c|c|c|c|c|}
\hline $\begin{array}{l}\text { Biênio } \\
(\mathrm{N})\end{array}$ & $\begin{array}{c}\text { Idade } \\
\text { média (dp) }\end{array}$ & $\begin{array}{c}\text { Sexo } \\
\text { Homens (\%) }\end{array}$ & $\begin{array}{l}\text { Naturalidade } \\
\text { DF }(\%)\end{array}$ & $\begin{array}{l}\text { Preferência por } \\
\left.\text { carreira* }^{*} \%\right)\end{array}$ & $\begin{array}{l}\text { Acesso no } 1^{\circ} \\
\text { semestre }(\%)\end{array}$ \\
\hline $1^{\mathrm{o}}(105)$ & $19,1(2,8)$ & 50,5 & 38,1 & 81,9 & 49,5 \\
\hline $2^{\circ}(107)$ & $19,6(3,8)$ & 51,4 & 32,7 & 72,9 & 54,2 \\
\hline $3^{\mathrm{o}}(117)$ & $19,4(3,0)$ & 56,4 & 50,4 & 81,2 & 47,9 \\
\hline $4^{\circ}(142)$ & $19,3(2,4)$ & 61,3 & 45,1 & 76,1 & 45,1 \\
\hline $5^{\circ}(138)$ & $19,6(2,7)$ & 56,5 & 57,2 & 75,4 & 50,7 \\
\hline Total (609) & $19,4(2,9)$ & 55,7 & 45,4 & 77,3 & 49,3 \\
\hline
\end{tabular}

* Inclui preferência principal por Cirurgia ou Clínica Médica ou Pediatria.

A Tabela 2 mostra as médias dos escores do QVC, intenção de aprender, autoconfiança como aprendiz e rendimento acadêmico entre os cinco grupos de biênio de ingresso. $\mathrm{Na}$ comparação de médias, as diferenças foram significantes ape- nas para autoconfiança, em que se observou índice mais elevado para os três biênios mais recentes em comparação com os dois primeiros (Anova, $\mathrm{df}=4 / 602 ; \mathrm{F}=6,7 ; \mathrm{P}<0,001$; teste post-hoc R-E-G-W Q).

TABELA 2

Comparação de médias (dp) de autoconfiança como aprendiz, intenção de aprender, rendimento cognitivo no primeiro ano e escore do Questionário de Valoração do Curso (QVC) entre alunos de Medicina agrupados segundo o biênio de ingresso no curso

\begin{tabular}{lcccc}
\hline Biênio (N) & $\begin{array}{c}\text { Intenção de aprender } \\
\text { média (dp) }\end{array}$ & $\begin{array}{c}\text { Autoconfiança } \\
\text { média }(\mathrm{dp})\end{array}$ & $\begin{array}{c}\text { Rendimento } \\
\text { média (dp) }\end{array}$ & $\begin{array}{c}\text { QVC } \\
\text { média (dp) }\end{array}$ \\
\hline $1^{\mathrm{o}}(103)$ & $85,9(11,4)$ & $76,8(18,4)$ & $3,72(0,51)$ & $116,8(13,0)$ \\
$2^{\mathrm{o}}(107)$ & $85,7(14,7)$ & $77,8(16,0)$ & $3,74(0,62)$ & $119,0(11,8)$ \\
$3^{\mathrm{o}}(117)$ & $86,9(11,9)$ & $83,6(15,4)$ & $3,80(0,61)$ & $118,7(13,2)$ \\
$4^{\mathrm{o}}(142)$ & $86,9(12,7)$ & $85,3(14,4)$ & $3,62(0,49)$ & $117,0(13,2)$ \\
$5^{\mathrm{o}}(138)$ & $87,5(11,6)$ & $83,6(16,4)$ & $3,66(0,53)$ & $117,7(12,5)$ \\
\hline Total $(607)$ & $87,0(12,5)$ & $81,8(16,3)$ & $3,70(0,55)$ & $117,8(12,8)$ \\
\hline
\end{tabular}

Não houve diferenças significantes dos escores do QVC entre os alunos agrupados por turmas (Anova, df = 19/587; $\mathrm{F}=1,50 ; \mathrm{P}=0,078)$. A média foi $117,85(\mathrm{dp}=12,79)$ na totali- dade de 607 participantes. Dentre esses, 56,3\% mostraram escores acima de 117 (> 75\% na faixa de pontuação), enquanto para 3,0\% os escores não ultrapassaram 90 (50\% na faixa 
de pontuação). Três itens do QVC mostraram as mais fortes correlações (corrigidas) com o escore total:

Item 20: O curso até aqui foi uma vivência de aprendizagem construtiva e definitivamente proveitosa; $r=$ 0,613 ;

Item 26: As disciplinas cursadas não tiveram impacto no meu entendimento do que sou e do quero; $r=0,608$ (polaridade invertida);

Item 13: O curso de Medicina tem sido uma vivência de aprendizagem gratificante para mim; $r=0,597$.

As pontuações mais elevadas entre os 36 itens foram registradas para os três seguintes:

Item 01: O curso de Medicina tem sido uma vivência de aprendizagem muito valiosa para mim; média $=3,72$ $(\mathrm{dp}=0,46)$;

Item 34: Seguir medicina fez pouca diferença para mim até aqui; média $=3,70(\mathrm{dp}=0,57)$. Polaridade invertida;

Item 03: Considero minha vivência de aprendizagem no curso de Medicina um tempo e esforço bem empregados; média $=3,64(\mathrm{dp}=0,53)$.
Em contraposição, os três itens com pontuação mais baixa foram os seguintes:

Item 19: Entendo melhor como os outros me percebem; média $=2,78(\mathrm{dp}=0,69)$;

Item 12: As disciplinas cursadas me ajudaram a perceber a importância de meus próprios sentimentos; média = 2,90 (dp $=0,73)$;

Item 24: Lidei, de algum modo, com mais desafios nas disciplinas do curso e estou satisfeito por isso; média $=2,91$ $(\mathrm{dp}=0,77)$.

A Tabela 3 revela diferenças nos escores do QVC entre os participantes agrupados por sexo, ou naturalidade, ou semestre de acesso ao curso, ou preferência inicial por carreira. Quatro grupos de classificação (sexo feminino, nascimento fora do DF, acesso no $1^{\circ}$ semestre, preferência por carreiras mais populares) mostraram escores mais elevados do QVC do que aqueles dos respectivos grupos alternativos (testes $\mathbf{t}$, maior $\mathrm{P}$ $=0,044)$. A diferença foi maior e mais significante $(\mathrm{P}<0,001)$ na comparação de QVC entre grupos de preferência por carreira. Foi menor e pouco significante $(P=0,044)$ na comparação entre grupos de naturalidade.

TABELA 3

Comparação de médias (dp) do escore do Questionário de Valoração do Curso (QVC) entre alunos de Medicina agrupados por sexo, naturalidade, semestre de acesso ao curso e preferência inicial por carreira (testes $t$ )

\begin{tabular}{|c|c|c|c|c|}
\hline Fatores & $\frac{\text { QVC }}{\text { média (dp) }}$ & Diferença & $\mathbf{t}$ & $\mathrm{P}$ \\
\hline Sexo & & 2,52 & 2,46 & 0,014 \\
\hline Feminino $(n=269)$ & $119,25(11,63)$ & & & \\
\hline Masculino $(\mathrm{n}=338)$ & $116,73(13,55)$ & & & \\
\hline Naturalidade & & $-2,21$ & $-2,12$ & 0,034 \\
\hline Distrito Federal $(\mathrm{n}=276)$ & $116,64(12,91)$ & & & \\
\hline Outra unidade $(\mathrm{n}=331)$ & $118,85(12,62)$ & & & \\
\hline Acesso ao curso & & 2,47 & 2,49 & 0,013 \\
\hline $1^{\circ}$ semestre $(n=300)$ & $119,15(12,03)$ & & & \\
\hline $2^{\circ}$ semestre $(\mathrm{n}=307)$ & $116,57(13,39)$ & & & \\
\hline Preferência por carreira & & 5,58 & 4,53 & $<0,001$ \\
\hline Opção $\mathrm{MCP}^{*}(\mathrm{n}=471)$ & $119,14(12,29)$ & & & \\
\hline Outra opção $(n=136)$ & $113,59(13,33)$ & & & \\
\hline
\end{tabular}

*Clínica Médica ou Cirurgia ou Pediatria.

A contribuição relativa dos diferentes fatores para a variabilidade do escore do QVC foi analisada mediante o procedimento unianova do Modelo Linear Geral. A Tabela 4 mostra os efeitos (between-subjects) principais. Nove fontes de variação (incluindo duas interações entre variáveis) explicam em conjunto $24 \%$ da variabilidade do QVC expressa no teste eta ${ }^{2}$. As parcelas mais importantes na atribuição da variabilidade, em ordem decrescente de magnitude de efeito, derivam de intenção de aprender, autoconfiança como aprendiz e preferência por carreira. Aquelas menos expressivas derivam de naturalidade, sexo e rendimento no primeiro ano, na mesma ordem. A sétima variável, semestre de acesso, mostrou um efeito intermediário entre os outros fatores citados. Além disso, observou-se interação entre naturalidade e sexo, assim como entre naturalidade e semestre de acesso. Estudantes de sexo feminino, nascidas noutros estados, mostraram escores de QVC significantemente maiores do que seus pares masculinos de outros estados; uma diferença inversa, mas não significante, foi observada entre os naturais do Distrito Federal. 
Em acréscimo, observou-se que estudantes com acesso no primeiro semestre, entre os naturais do Distrito Federal, mostraram escores de QVC significantemente maiores do que aqueles com acesso no segundo semestre; uma diferença inversa, mas não significante, foi observada entres os nascidos noutros estados.

\section{TABELA 4}

Modelo Linear Geral, Unianova. Teste de efeitos (between-subjects) de fatores (sexo, naturalidade, semestre de acesso, categoria de preferência por carreira), ajustados para co-variáveis (autoconfiança, intenção de aprender e rendimento cognitivo no primeiro ano) no escore do Questionário de Valoração do Curso (QVC), em alunos de Medicina $(\mathrm{N}=607)$

\begin{tabular}{lccccc}
\hline Fonte de variação & $\begin{array}{c}\text { Soma de } \\
\text { quadrados (SQ) }\end{array}$ & $\mathrm{df}$ & $\begin{array}{c}\text { Média } \\
(\mathrm{MQ})\end{array}$ & $\mathrm{F}$ & Significância \\
\hline Modelo corrigido & $24.221,2$ & 9 & $2.691,2$ & 21,4 & $<0,001$ \\
Intercepto & $30.998,1$ & 1 & $30.998,1$ & 247,0 & $<0,001$ \\
Sexo & 506,8 & 1 & 506,8 & 4,0 & 0,045 \\
Naturalidade & 553,6 & 1 & 553,6 & 4,4 & 0,036 \\
Preferência por carreira & $1.610,8$ & 1 & $1.610,8$ & 12,8 & $<0,001$ \\
Semestre de acesso & 969,8 & 1 & 969,8 & 7,7 & 0,006 \\
Intenção de aprender & $9.775,7$ & 1 & $9.775,7$ & 77,9 & $<0,001$ \\
Autoconfiança & $2.329,5$ & 1 & $2.329,5$ & 18,6 & $<0,001$ \\
Rendimento no 10 $1^{\text {ano }}$ & 361,8 & 1 & 361,8 & 2,9 & 0,90 \\
Sexo*naturalidade & 583,0 & 1 & 583,0 & 4,6 & 0,032 \\
Acesso*naturalidade & $1.375,0$ & 1 & $1.375,0$ & 10,9 & 0,001 \\
Erro & $74.910,3$ & 597 & 125,5 & & \\
Total & $8.528 .830,0$ & 607 & & & \\
Total corrigido & $99.131,4$ & 606 & & & \\
\hline
\end{tabular}

Variável de desfecho: escore total do Questionário de Valoração do Aprendizado, referente ao primeiro ano do curso. Coeficiente de determinação $\left(R^{2}\right)$ ajustado: 0,233

A Tabela 5 realça os coeficientes de correlação do escore do QVC com medidas subjetivas e objetivas em duas perspectivas: transversal (intenção para aprender, autoconfiança como aprendiz e rendimento cognitivo no primeiro ano) e prospectiva (número de monitorias em quatro semestres e rendimento cognitivo no sexto período do curso). As correlações foram significantes para os cincos pares de variáveis; as associações mais fortes foram observadas entre o QVC e as medidas subjetivas de intenção de aprender $(r=0,388)$ e autoconfiança como aprendiz $(r=0,241)$. Embora claramente significante, a correlação entre QVC e atividade de monitoria foi pequena $(r=0,143)$.

TABELA 5

Coeficientes de correlação entre escores do Questionário de Valoração do Curso (QVC) e medidas de atitudes na aprendizagem e de sucesso acadêmico de alunos de Medicina

\begin{tabular}{|c|c|c|c|}
\hline & & QVC & \\
\hline Medidas & $\mathrm{N}$ & $\mathbf{r}$ & $\mathbf{P}$ \\
\hline \multicolumn{4}{|l|}{ Componente transversal } \\
\hline Autoconfiança como aprendiz & 607 & 0,241 & $<0,001$ \\
\hline Intenção de aprender & 607 & 0,393 & $<0,001$ \\
\hline Rendimento cognitivo $-1^{\mathrm{o}}$ ano & 607 & 0,134 & 0,001 \\
\hline \multicolumn{4}{|l|}{ Componente prospectivo } \\
\hline$N^{\circ}$ de monitorias ( $2^{\circ}$ ao $5^{\circ}$ semestres) & 592 & 0,143 & $<0,001$ \\
\hline Rendimento cognitivo- $6^{\circ}$ semestre & 578 & 0,090 & 0,030 \\
\hline
\end{tabular}




\section{DISCUSSÃO}

Ao longo do decênio do estudo, o uso do Questionário de Valoração do Curso favoreceu a participação universal dos alunos de Medicina numa reflexão sobre a vivência inicial no curso, em razão do interesse despertado pelo conteúdo e da simplicidade no seu preenchimento. As características das respostas ao questionário (incluindo o nível de consistência interna e o fator subjacente preponderante) indicam que o instrumento detecta predominantemente um efeito global nos estudantes, o qual provavelmente expressa o conjunto de características da ambiência educacional. O escore médio do QVC sintetiza esse efeito. A comparação dos escores dos QVC entre formados e não formados sugere que um valor baixo desse índice é um fator de risco de evasão, independente do rendimento cognitivo.

No contexto deste estudo, a ausência de tendência temporal ou de diferenças significantes nos escores entre turmas agregadas ou não sugere que a variância nesse índice do QVC decorreu mais de diferenças nas percepções pessoais dos participantes e bem menos de mudanças impactantes ou sustentáveis ocorridas na organização das disciplinas ou das práticas docentes ao longo do decênio.

A magnitude da variabilidade do escore do QVC, decorrente da natureza das percepções individuais da vivência do primeiro ano do curso, foi expressa nos resultados da análise unianova. Três fatores de contribuição mais expressiva são realçados: os efeitos de preferência por carreira, autoconfiança como aprendiz e intenção de aprender. O tamanho do efeito da preferência por carreira foi inesperado, mas consistente com a idéia do papel da configuração individual das atitudes e expectativas na aprendizagem - geradas, no caso, pela orientação profissional - sobre a valoração pessoal do aprendizado.

A medida da autoconfiança como aprendiz serve como indicador de eficácia pessoal ou senso de competência na aprendizagem ${ }^{9}$. Uma associação positiva e significante entre o nível de autoconfiança e o índice do QVC tem sido observada consistentemente e provavelmente reflete a percepção de segurança no aprendizado cognitivo ${ }^{6}$.

A importância da contribuição da intenção de aprender (traduzindo o grau de motivação para realizar os esforços requeridos) deve ser ressaltada por sua coerência com achados anteriores que sinalizaram o efeito preditivo significante do escore do QVC em relação ao grau de motivação para prosseguir os estudos ${ }^{6}$. Evidências de novo trabalho' ${ }^{12}$ (concluído, mas não publicado) apontam para uma associação substancial entre o índice do QVC e a autodeterminação da motivação em estudantes de Medicina.
Os achados atuais e anteriores sugerem uma contribuição importante para a variabilidade do escore do QVC de múltiplos elementos do contexto interno do aprendiz, tais como intenção de aprender, autoconfiança, alvo de carreira, bem como a motivação autônoma e orientação profunda e reflexiva que caracterizam a aprendizagem significativa. Esses elementos interagem com os componentes físico, cognitivo e afetivo do contexto educacional, na concepção de Koens ${ }^{13}$, para definir o valor somativo na apreciação da vivência. Nesse sentido, o escore do QVC representa um índice de valoração do aprendizado subjetivo e pessoal realizado nas circunstâncias do contexto educacional.

O caráter multidimensional do espectro de respostas ao QVC possivelmente explica a relação entre o índice de valoração do aprendizado referente ao primeiro ano de estudos e o número de monitorias acumulado nos dois anos subseqüentes. A atividade de monitoria representa uma medida da adesão aos valores e princípios do curso e, conseqüentemente, do sucesso acadêmico, embora sua procura se deva a razões diversas, que incluem a busca de orientação profissional e de desenvolvimento de habilidades, e também de recompensas extrínsecas.

Em suma, os achados do estudo e de outros relatos publicados reafirmam a fidedignidade do Questionário de Valoração do Curso na apreciação do efeito global da vivência curricular, bem como da variabilidade das respostas, atribuível à própria diversidade na composição do alunado. Essa diversidade expressa em parte a configuração individual das atitudes e expectativas na aprendizagem.

Concluindo, as respostas ao QVC refletem a perspectiva de valoração do aprendizado pessoal na interação com as dimensões do contexto educacional. O instrumento tem, portanto, utilidade no acompanhamento pedagógico da iniciação profissional.

\section{AGRADECIMENTOS}

O autor é grato aos alunos pela genuína cooperação, bem como aos ex-diretores Eduardo Queiroz, Josimar França e Paulina de Freitas Targino pelo apoio continuado.

\section{REFERÊNCIAS}

1. Genn JM. AMEE medical education guide $\mathrm{n}^{\circ} 23$ (part 1): curriculum, environment, climate, quality and change in medical education: a unifying perspective. Med Teach. 2001; 23(4): 337-44.

2. Genn JM. AMEE medical education guide $\mathrm{n}^{\circ} 23$ (part 2): curriculum, environment, climate, quality and change in 
medical education: a unifying perspective. Med Teach. 2001; 23(5): 445-54.

3. Nehari M, Bender H. Meaningfulness of a learning experience: a measure for educational outcomes in higher education. Higher Educ. 1978; 7(1): 1-11.

4. Sobral DT. The problem-based learning approach as an enhancement factor of personal meaningfulness of learning. Higher Educ. 1995; 29(1): 93-101.

5. Sobral DT. Efeitos da mudança curricular: características do aprendiz e desfechos da aprendizagem. Rev Bras Educ Med. 1996; 20(1): 15-20.

6. Sobral DT. Medical students' self-appraisal of first-year learning outcomes: use of the course valuing inventory. Med Teach. 2004; 26(3): 234-8.

7. Roff S, McAleer S, Harden RM, et al. Development and validation of the Dundee Ready Education Environment Measure (DREEM). Med Teach. 1997;19: 295-9.

8. Hulley SB, Cummings SR, eds. Designing clinical research. Baltimore: Williams \& Wilkins; 1988.

9. Schunk DH, Pajares F. Self-efficacy in education revisited: empirical and applied evidence. In: McInerney DM, Van Etten S, eds. Big theories revisited. Greenwich: Information Age; 2004. p.115-138.

10. Fraenkel JR, Wallen NE. How to design and evaluate research in education. $5^{\text {th }}$ ed. Boston: McGraw-Hill; 2003.

11. Howell DC. Statistical methods for psychology. $5^{\text {th }}$ ed. Pacific Grove: Duxbury; 2002.

12. Sobral DT. Autodeterminação da motivação em alunos de medicina: relações com motivos de escolha da opção e intenção de adesão ao curso. Rev Bras Educ Med. 2008. (no prelo)

13. Koens F, Mann KV, Custers EJFM, Cate OTJT. Analysing the concept of context in medical education. Med Educ. 2005; 39(12): 1243-9.

\section{CONFLITOS DE INTERESSE:}

Declarou não haver.

\section{ENDEREÇO PARA CORRESPONDÊNCIA}

Dejano T. Sobral

Universidade de Brasília

Faculdade de Medicina - C.P. 04569

70919-970 - Brasília - DF

E-mail: dtsobral@unb.br

\section{ANEXO}

\section{QUESTIONÁRIO DE VALORAÇÃO DO CURSO*}

Valor da Vivência. Expresso, a seguir, minha percepção do valor e do significado do aprendizado já realizado no curso de Medicina, marcando cada item abaixo assim:

$\mathrm{CT}=$ Concordo Totalmente, $\mathrm{C}=$ Concordo, $\mathrm{D}=$ Discordo, DT $=$ Discordo Totalmente.

1. O curso de Medicina tem sido uma vivência de aprendizagem muito valiosa para mim.

2. Tal vivência de aprendizagem ajudou-me a ficar mais consciente de meus sentimentos e reações.

3. Considero minha vivência de aprendizagem no curso de Medicina um tempo e esforço bem empregados.

4. As disciplina do curso não tiveram impacto positivo no meu desenvolvimento pessoal.

5. As disciplinas anteriores me ajudaram a adquirir conhecimentos básicos importantes.

6. Tais disciplinas não tiveram impacto no jeito em que eu me comunico.

7. Posso agora lidar com as matérias do curso médico de uma perspectiva mais ampla.

8. No curso de Medicina ainda não desenvolvi minhas próprias metas de aprendizado.

9. Tenho trabalhado, de certa forma, com mais empenho nas disciplinas do curso do que fazia habitualmente antes.

10. Minha vivência prévia de aprendizagem no curso médico não foi significativa.

11. Não adquiri muita informação nas disciplinas já cursadas.

12. Tais disciplinas me ajudaram a perceber a importância de meus próprios sentimentos.

13. O curso tem sido uma vivência de aprendizagem gratificante para mim.

14. As disciplinas anteriores foram úteis auxiliando-me a desenvolver novos modos de aprender.

15. Sinto-me agora mais perceptivo e sensível às necessidades dos outros.

16. As disciplinas do curso não têm sido estimulantes.

17. Tenho sido, de certa forma, mais acessível e compartilhativo em virtude das vivências do curso de Medicina. 
18. Estou mais capacitado a interpretar os problemas apresentados nasdisciplinas cursadas.

19. Entendo melhor como os outros me percebem.

20. O curso até aqui foi uma vivência de aprendizagem construtiva e definitivamente proveitosa.

21. Participei menos nas disciplinas do curso do que fazia habitualmente.

22. Sinto-me bem comigo mesmo, sob certos aspectos, devido às disciplinas já cursadas.

23. Meu entendimento das matérias cursadas não aumentou muito.

24. Lidei, de algum modo, com mais desafios nas disciplinas do curso e estou satisfeito por isso.

25. Gostaria de fazer outras disciplinas parecidas com as anteriores.

26. As disciplinas já cursadas não tiveram impacto no meu entendimento do que sou e do que quero.

27. As disciplinas prévias ajudaram-me a alcançar uma compreensão mais profunda da Medicina.
28. Não li, nem pensei mais do que se esperava realmente.

29. As disciplinas prévias não me ajudaram a adquirir um conhecimento cuidadoso das matérias.

30. Alguns dos meus valores foram clarificados devido a essa vivência de aprendizagem no curso médico.

31. Eu não recomendaria meu curso a um amigo, até aqui.

32. Tenho agora uma noção mais clara e integrada das matérias do curso.

33. Penso que aprendi a ser mais tolerante.

34. Seguir Medicina fez pouca diferença para mim até aqui.

35. Não me foi possível interligar as noções e fazer muito sentido do conteúdo.nas disciplinas anteriores.

36. Nas disciplinas já cursadas assumi mais responsabilidade por minha própria aprendizagem do que fazia anteriormente.

* Versão, adaptada pelo autor, do Course Valuing Inventory33, com autorização do Dr Hilary Bender, Boston University. 